\title{
A checklist for the zooplankton of the Middle Xingu - an Amazon River system
}

\author{
Brito, SAC. ${ }^{a}$, Camargo, M. ${ }^{b *}$, Melo, NFAC. ${ }^{c}$ and Estupiñan, $R A .^{b}$ \\ ${ }^{\mathrm{a}}$ Governo do Pará, Secretaria de Estado de Educação - SEDUC, \\ Rodovia Augusto Montenegro, Km 10, Icoaraci, Belém, PA, Brazil \\ ' Instituto Federal de Educação Ciência e Tecnologia da Paraíba - IFPB, Campus Cabedelo, \\ Rua Santa Rita de Cássia, s/n, Jardim Camboinha, CEP 58103-772, Cabedelo, PB, Brazil \\ 'Laboratório de Ecologia Aquática e Aqüicultura Tropical, Universidade Federal Rural da Amazônia - UFRA, \\ Avenida Perimetral, 2501, Terra Firme, CEP 66077-530, Belém, PA, Brazil \\ *e-mail: camargo.zorro@gmail.com
}

Received: March 11, 2015 - Accepted: May 22, 2015 - Distributed: August 31, 2015

(With 1 figure)

\begin{abstract}
A zooplankton checklist is presented for the Middle Xingu River, based on surveys conducted at four sites in the main channel and two fluvial lakes. A total of 175 taxa are listed, including 141 rotifers, 20 cladocerans, and five copepods. Rapids presented the greatest species richness, with up to 124 taxa, while Ilha Grande lake had 70 taxa, the lowest number. Non-planktonic benthic larvae were recorded frequently in the samples.
\end{abstract}

Keywords: clear water rivers, zooplankton, fluvial habitats, limnology.

\section{Diversidade zooplanctônica do médio Rio Xingu - bacia amazônica}

\section{Resumo}

Levantamentos realizados em dois lagos e no canal principal do médio Xingu objetivaram a listar a diversidade do zooplâncton. De um total de 175 táxons, 141 foram rotíferos, 20 cladóceros e 5 copépodes. As corredeiras foram os ambientes mais diversos com 124 táxons, enquanto que no Lago da Ilha Grande se registraram somente setenta táxons. Destaca-se a ocorrência de grupos de hábito não planctônico na coluna d'água do rio que indica o efeito perturbador da correnteza.

Palavras-chave: águas claras amazônicas, zooplâncton, ambientes fluviais, limnologia.

\section{Introduction}

Many inventories of the zooplankton diversity of the rivers of the Amazon basin are available, especially for the principal, white- and black-water tributaries. The principal studies have focused on the marginal várzea lakes of the Amazon and Negro floodplains (see Robertson and Hardy, 1984; Koste and Robertson, 1990; Brandorff, 1973; Brandorff and Andrade, 1978; Koste and Robertson, 1983; Carvalho, 1983; Hardy, 1980; Hardy et al., 1984; Robertson and Hardy, 1984; Santos-Silva et al., 1989; Waichman et al., 2002). However, studies of the composition of the zooplankton of clear-water rivers are scarce. The available studies include those of Bozelli $(1992,1994)$ and Bozelli et al. (2000) in the region of the Trombetas River, Koste (1972, 1974 a,b, 1989) on the Tapajós, and Brandorff et al. (1982) on the lower Nhamundá River. Given this, the present study provides a checklist of the zooplankton of two fluvial lakes and four sites in the main channel of the middle Xingu, a clear-water river in the southeastern Amazon basin.

\section{Material and Methods}

\subsection{Study area}

The present study was conducted along a $180 \mathrm{~km}$ stretch of the middle Xingu River in Pará, in the eastern Brazilian Amazon basin (Figure 1). This region is characterized by numerous waterfalls and rapids, as well as extensive areas of alluvial rainforest (Camargo et al., 2005).

The climate of the study area is of Köppen's A type, with variants Aw and Am (Critchfield, 1968). Mean annual temperatures in the study area oscillate between $17.5^{\circ} \mathrm{C}$ and $24.5{ }^{\circ} \mathrm{C}$, with relative humidity of $84-86 \%$. Mean annual precipitation varies from $2066.8 \mathrm{~mm}$ to $2379.4 \mathrm{~mm}$ (Camargo et al., 2005).

Six environments were monitored in the present study. Two were located in lakes on river islands (Ilha Grande and Pimentel) and the other four (Boa Esperança, Arroz Cru, Caitucá and CNEC) in the main Xingu River. Ilha 
Grande lake $\left(3^{\circ} 34^{\prime} 47^{\prime \prime} \mathrm{S}, 52^{\circ} 23^{\prime} 42^{\prime \prime} \mathrm{W}\right)$ is semi-circular, with a depth of $0.5-2.5 \mathrm{~m}$, and a total area of approximately $15,612 \mathrm{~m}^{2}$. The bed of this lake is covered in slimy silt, sand, and leaf litter derived from the island's dense alluvial rainforest, and high concentrations of phytoplankton were associated with the reduced Secchi transparency (0.8-1.1 m) and high levels of dissolved oxygen (DO: 5.0$\left.7.5 \mathrm{mg} .1^{-1}\right)$, and the slightly acid water $(\mathrm{pH}=5.9-6.7)$ with low conductivity, of $30 \mathrm{uS} . \mathrm{cm}^{-1}$ (Estupiñan and Camargo, 2008). The second lake, Pimentel ( $3^{\circ} 25^{\prime} 46^{\prime \prime} \mathrm{S}, 52^{\circ} 24^{\prime} 4^{\prime \prime}$ $\mathrm{W})$, located on the river's Great Bend, is elliptical in shape, with a mean surface area of $1570 \mathrm{~m}^{2}$, depths of between 0.8 and $3.0 \mathrm{~m}$, with low $\mathrm{pH}(5.0-5.4)$ and Secchi transparency $(0.900 .98 \mathrm{~m})$, and low DO (2 mg.L-1 $)$ and reduced levels of chlorophyll, which determine its low levels of primary productivity (Estupiñan and Camargo, 2008). During the rainy season, the forest is flooded by the creeks that drain the area surrounding the lakes.

On the river margin, Boa Esperança $\left(3^{\circ} 34^{\prime} 46^{\prime \prime} \mathrm{S}\right.$, $52^{\circ} 24^{\prime} 42^{\prime \prime} \mathrm{W}$ ) is located in the vicinity of Ilha Grande

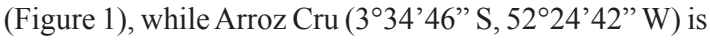
near Pimentel Island. The Caitucá marginal site ( $3^{\circ} 33^{\prime} 47^{\prime \prime} \mathrm{S}$, $51^{\circ} 24^{\prime} 42^{\prime \prime} \mathrm{W}$ ) is located in the large river bend (Cotubelo) deflection (Estupinãn and Camargo, 2008). The CNEC site $\left(3^{\circ} 16^{\prime} 16^{\prime \prime} \mathrm{S}, 51^{\circ} 24^{\prime} 42^{\prime \prime} \mathrm{W}\right)$ is upstream from the larger waterfalls before the lower Xingu River. The $\mathrm{pH}$ of the river varied from 6.4 to 7.6 and conductivity was low (20-31 uS.cm $\left.{ }^{-1}\right)$ which, together with the DO concentrations of 7.1-8.6 mg. $\mathrm{L}^{-1}$ indicate highly that the water is highly oxygenated (Estupinãn and Camargo, 2008).

Every two months between August 2006 and June 2007, four zooplankton samples were collected at the surface and middle depths of the two lakes (Ilha Grande,
Pimentel), while two angular sub-surface samples were collected from four stretches of the main channel of the Xingu River (Figure 1). The samples were collected with a $40 \mu$ mesh plankton net equipped with a digital flowmeter in the river and by filtering 400 liters of water into a bucket in the lakes. The specimens collected were fixed and preserved in $4 \%$ formalin. Sorting and identification of specimens were carried out with a Wild-Leitz stereo-zoom dissecting microscope. Identification of the specimens was based on Cipólli and Carvalho (1973), Koste (1978), Paggi (1979), Robertson (1980), Brandorff et al. (1982), Koste and Robertson (1983), Koste et al. (1984), Reid (1985), Magalhães et al. (1988), Robertson et al. (1989), Santos-Silva et al. (1989), Koste and Robertson (1990), Korovchinsky (1992), Smirnov (1992), Paggi (1995), Segers (1995), Elmoor-Loureiro (1997), and Fernando (2002).

\section{Results}

This study recorded a total of 175 taxa, comprising 141 rotifers (80.6\%), 20 cladocerans (11.4\%), five copepods $(2.9 \%)$, and nine $(5.1 \%)$ bottom-dwelling protozoans, gastrotrichs and insects (Tables 1 and 2). In general, the fluvial habitats were the most diverse. The richness of taxa varied from 70 in Ilha Grande lake to 124 in the rapids. Despite being much smaller than Ilha Grande lake, Pimentel lake had a relatively high richness, with a total of 114 taxa.

The Rotifera was the most diverse group, accounting for approximately $80 \%$ of total taxon richness in each of the study environments (Table 1). Much less diverse were the Cladocera, with around $8 \%$ of the taxa, and the Calanoida and Copepoda, each with approximately $2 \%$ (Table 1 ).

Most of the rotifers (19.86\%) belonged to the family Lecanidae. Brachionus calyciflorus, Pallas, 1866; Testudinela

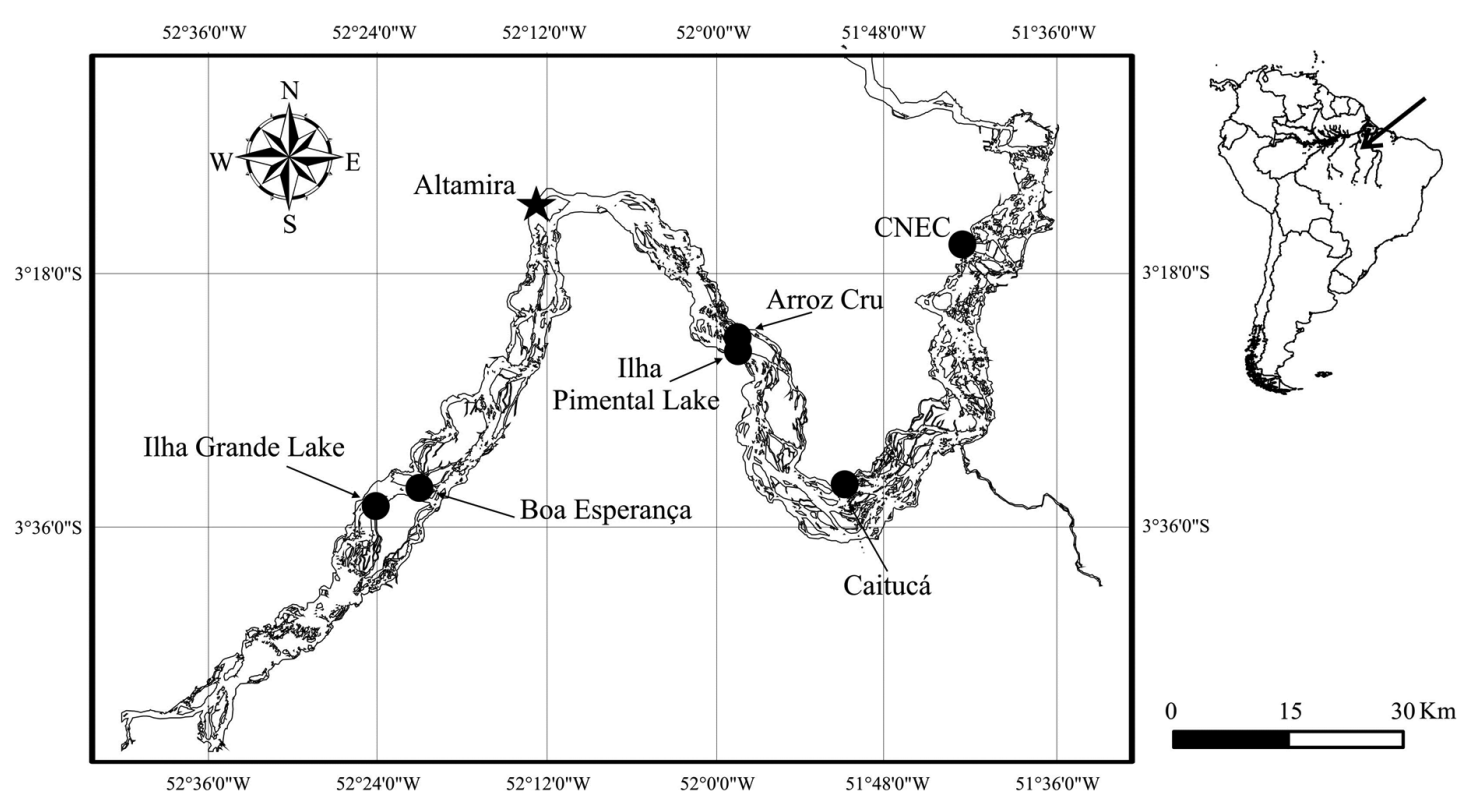

Figure 1. Map of the region with the localities in the main channel and island lakes. 
Table 1. Zooplankton species recorded in different environments of the Middle Xingu River (2006-2007).

\begin{tabular}{|c|c|c|c|c|c|}
\hline \multirow[b]{3}{*}{ Group } & \multirow[b]{3}{*}{ Habitat } & \multicolumn{4}{|c|}{ Environments and habitats } \\
\hline & & Islan & lakes & Main & hannel \\
\hline & & $\begin{array}{c}\text { Ilha } \\
\text { Grande }\end{array}$ & Pimental & Rapids & $\begin{array}{c}\text { Flow } \\
\text { restricted } \\
\text { waters }\end{array}$ \\
\hline
\end{tabular}

\section{ROTIFERA}

GASTROPODIDAE

\begin{tabular}{|c|c|c|c|c|c|}
\hline Ascomorpha ecaudis (Perty, 1850) & $\mathrm{Pl}$ & & $\mathrm{X}$ & $\mathrm{X}$ & \\
\hline Ascomorpha saltans (Bartsch, 1870) & $\mathrm{Pl}$ & $\mathrm{X}$ & $\mathrm{X}$ & & \\
\hline Ascomorpha sp. & $\mathrm{Pl}$ & & $\mathrm{X}$ & & \\
\hline Gastropus hyptopus (Ehrenberg, 1838) & NDT & & $\mathrm{X}$ & & $\mathrm{X}$ \\
\hline Gastropus stylifer Imhof, 1891 & NDT & $\mathrm{X}$ & & & \\
\hline Gastropus sp. & NDT & $\mathrm{X}$ & & & \\
\hline
\end{tabular}

\begin{tabular}{|c|c|c|c|c|c|}
\hline \multicolumn{6}{|l|}{ ASPLANCHNIDAE } \\
\hline Asplanchna priodonta Gosse, 1850 & $\mathrm{Pl}$ & $\mathrm{X}$ & & $\mathrm{X}$ & \\
\hline Asplanchna sieboldi (Leydig, 1845) & $\mathrm{Pl}$ & & $\mathrm{X}$ & & \\
\hline Asplanchna silvestri Daday, 1902 & $\mathrm{Pl}$ & & $\mathrm{X}$ & & \\
\hline Asplanchna sp. & $\mathrm{Pl}$ & $\mathrm{X}$ & $\mathrm{X}$ & $\mathrm{X}$ & $\mathrm{X}$ \\
\hline Harringia sp. & NDT & $\mathrm{X}$ & & & \\
\hline \multicolumn{6}{|l|}{ BRACHIONIDAE } \\
\hline Anuraeopsis fissa (Gosse, 1851) & $\mathrm{Pl}$ & $\mathrm{X}$ & $\mathrm{X}$ & $\mathrm{X}$ & $\mathrm{X}$ \\
\hline Anuraeopsis navicula Rousselet, 1910 & $\mathrm{Pl}$ & $\mathrm{X}$ & $\mathrm{X}$ & $\mathrm{X}$ & \\
\hline Anuraeopsis siolli Koste, 1972 & $\mathrm{Pl}$ & & $\mathrm{X}$ & & \\
\hline Brachionus ahlstromi (Lindeman, 1939) & $\mathrm{Pl}$ & & & $\mathrm{X}$ & $\mathrm{X}$ \\
\hline Brachionus angularis Gosse, 1851 & $\mathrm{Pl}$ & $\mathrm{X}$ & $\mathrm{X}$ & $\mathrm{X}$ & $\mathrm{X}$ \\
\hline Brachionus calyciflorus Pallas, 1866 & Pl & & & $\mathrm{X}$ & $\mathrm{X}$ \\
\hline Brachionus caudatus Barrois and Daday, 1894 & $\mathrm{Pl}$ & $\mathrm{X}$ & $\mathrm{X}$ & $\mathrm{X}$ & $\mathrm{X}$ \\
\hline Brachionus dolabratus Harring, 1915 & $\mathrm{Pl}$ & $\mathrm{X}$ & $\mathrm{X}$ & $\mathrm{X}$ & $\mathrm{X}$ \\
\hline Brachionus falcatus Zacharias, 1898 & $\mathrm{Pl}$ & $\mathrm{X}$ & $\mathrm{X}$ & $\mathrm{X}$ & $\mathrm{X}$ \\
\hline Brachionus gessneri Hauer, 1956 & $\mathrm{Pl}$ & $\mathrm{X}$ & $\mathrm{X}$ & $\mathrm{X}$ & $\mathrm{X}$ \\
\hline Brachionus mirabilis (Daday, 1897) & $\mathrm{Pl}$ & & $\mathrm{X}$ & & \\
\hline Brachionus mirus Dady, 1905 & $\mathrm{Pl}$ & $\mathrm{X}$ & $\mathrm{X}$ & $\mathrm{X}$ & $\mathrm{X}$ \\
\hline Brachionus patullus (Müller, 1953) & $\mathrm{Pl}$ & & $\mathrm{X}$ & $\mathrm{X}$ & $\mathrm{X}$ \\
\hline Brachionus quadridentatus Hermann, 1783 & NP & $\mathrm{X}$ & $\mathrm{X}$ & $\mathrm{X}$ & $\mathrm{X}$ \\
\hline Brachionus zahniseri Ahlstrom, 1934 & $\mathrm{Pl}$ & $\mathrm{X}$ & $\mathrm{X}$ & $\mathrm{X}$ & $\mathrm{X}$ \\
\hline Keratella americana Carlin, 1943 & Pl & $\mathrm{X}$ & $\mathrm{X}$ & $\mathrm{X}$ & $\mathrm{X}$ \\
\hline Keratella cochlearis Gosse, 1851 & $\mathrm{Pl}$ & $\mathrm{X}$ & $\mathrm{X}$ & $\mathrm{X}$ & $\mathrm{X}$ \\
\hline Keratella lenzi Hauer, 1953 & $\mathrm{Pl}$ & $\mathrm{X}$ & $\mathrm{X}$ & $\mathrm{X}$ & $\mathrm{X}$ \\
\hline Keratella nhamunda (Koste and Robertson, 1983) & $\mathrm{Pl}$ & & & & $\mathrm{X}$ \\
\hline Notholca sp. & NDT & & & $\mathrm{X}$ & \\
\hline Plathyias quadricornis Daday, 1905 & NP & & $\mathrm{X}$ & $\mathrm{X}$ & \\
\hline Squatinella sp. & NDT & & & & $\mathrm{X}$ \\
\hline \multicolumn{6}{|l|}{ NOTOMATIDAE } \\
\hline Cephalodella gibba (Ehrenberg, 1838) & NP & & $\mathrm{X}$ & $\mathrm{X}$ & \\
\hline Cephalodella intuta Myers, 1924 & NP & $\mathrm{X}$ & $\mathrm{X}$ & $\mathrm{X}$ & $\mathrm{X}$ \\
\hline Cephalodella mucronata Myers, 1924 & NP & & & & \\
\hline Cephalodella sp. & NP & & $\mathrm{X}$ & $\mathrm{X}$ & $\mathrm{X}$ \\
\hline \multicolumn{6}{|l|}{ COLLOTHECIDAE } \\
\hline Collotheca ambigua (Hudson, 1883) & NP & $\mathrm{X}$ & & & $\mathrm{X}$ \\
\hline Collotheca tenuilobata (Anderson, 1889) & NP & $\mathrm{X}$ & & $\mathrm{X}$ & $\mathrm{X}$ \\
\hline Collotheca sp1. & NDT & $\mathrm{X}$ & $\mathrm{X}$ & $\mathrm{X}$ & $\mathrm{X}$ \\
\hline Collotheca sp2. & NDT & & $\mathrm{X}$ & $\mathrm{X}$ & $\mathrm{X}$ \\
\hline
\end{tabular}

$\mathrm{Pl}=$ planktonic, $\mathrm{NP}=$ not planktonic, $\mathrm{NDT}=$ non determined 
Table 1. Continued...

\begin{tabular}{|c|c|c|c|c|c|}
\hline \multirow[b]{3}{*}{ Group } & \multirow[b]{3}{*}{ Habitat } & \multicolumn{4}{|c|}{ Environments and habitats } \\
\hline & & Islar & lakes & Mair & hannel \\
\hline & & $\begin{array}{c}\text { Ilha } \\
\text { Grande }\end{array}$ & Pimental & Rapids & $\begin{array}{c}\text { Flow } \\
\text { restricted } \\
\text { waters }\end{array}$ \\
\hline iatus (Goldfuss, 1820) & NP & & & $\mathrm{X}$ & $\mathrm{X}$ \\
\hline NOCHLIDAE & & & & & \\
\hline s (Hudson, 1875) & NDT & X & & & $\mathrm{X}$ \\
\hline
\end{tabular}

\begin{tabular}{|c|c|c|c|c|c|}
\hline Stephanoceros fimbriatus (Goldfuss, 1820) & NP & & & $\mathrm{X}$ & $\mathrm{X}$ \\
\hline \multicolumn{6}{|l|}{ CONOCHLIDAE } \\
\hline Conochilus dossuaris (Hudson, 1875) & NDT & $\mathrm{X}$ & & & $\mathrm{X}$ \\
\hline \multicolumn{6}{|l|}{ EPIPHANIDAE } \\
\hline Epiphanes clavatula (Ehrenberg, 1832) & $\mathrm{Pl}$ & & $\mathrm{X}$ & & \\
\hline Epiphanes macrourus (Barrois and Daday, 1894) & Pl & $\mathrm{X}$ & $\mathrm{X}$ & $\mathrm{X}$ & $\mathrm{X}$ \\
\hline Microcodides chlaena Gosse, 1886 & NDT & & $\mathrm{X}$ & $\mathrm{X}$ & \\
\hline
\end{tabular}

\begin{tabular}{|c|c|c|c|c|}
\hline Euchlanis dilatata Ehrenberg, 1832 & NP & & $\mathrm{X}$ & $\mathrm{X}$ \\
\hline Euchlanis incisa Carlin, 1939 & NP & $\mathrm{X}$ & & \\
\hline Dipleuchlanis propatula (Gosse, 1886) & NP & $\mathrm{X}$ & $\mathrm{X}$ & $\mathrm{X}$ \\
\hline
\end{tabular}

\begin{tabular}{lccccc}
\hline Filinia longiseta (Ehrenberg, 1834) & $\mathrm{Pl}$ & $\mathrm{X}$ & $\mathrm{X}$ & $\mathrm{X}$ & $\mathrm{X}$ \\
\hline Filinia opoliensis (Zacharias, 1898) & $\mathrm{Pl}$ & $\mathrm{X}$ & $\mathrm{X}$ & $\mathrm{X}$ & $\mathrm{X}$ \\
\hline Filinia pejleri Hutchinson, 1964 & $\mathrm{Pl}$ & $\mathrm{X}$ & $\mathrm{X}$ & $\mathrm{X}$ & $\mathrm{X}$ \\
\hline Filinia sp. & NDT & & $\mathrm{X}$ & $\mathrm{X}$ & \\
\hline
\end{tabular}

\section{HEXARTHRIDAE}

\begin{tabular}{cccccc}
\hline Hexarthra intermedia Wieszniewski, 1929 & Pl & X & X & X & X \\
\hline LECANIDAE & & & & & \\
\hline Lecane arcuata $($ Ryce, 1891). & NP & & X & &
\end{tabular}

\begin{tabular}{|c|c|c|c|c|c|}
\hline Lecane arcuata (Ryce, 1891). & NP & & $\mathrm{X}$ & & \\
\hline Lecane arculeata (Akubski, 1912). & NP & & & & $\mathrm{X}$ \\
\hline Lecane bulla (Gosse, 1886) & $\mathrm{Pl} / \mathrm{NP}$ & & $\mathrm{X}$ & $\mathrm{X}$ & $\mathrm{X}$ \\
\hline Lecane clara (Bryce, 1892$)$ & NP & & $\mathrm{X}$ & & \\
\hline Lecane closterocerca (Schmarda, 1856) & NP & $\mathrm{X}$ & $\mathrm{X}$ & $\mathrm{X}$ & $\mathrm{X}$ \\
\hline Lecane copeis (Harring and Myers, 1926) & NP & & & $\mathrm{X}$ & $\mathrm{X}$ \\
\hline Lecane curvicornis (Murray, 1913) & $\mathrm{Pl}$ & $\mathrm{X}$ & $\mathrm{X}$ & $\mathrm{X}$ & $\mathrm{X}$ \\
\hline Lecane hamata (Stockes, 1896) & NP & & $\mathrm{X}$ & $\mathrm{X}$ & $\mathrm{X}$ \\
\hline Lecane hornemanni (Ehrenberg, 1834) & NP & & & $\mathrm{X}$ & $\mathrm{X}$ \\
\hline Lecane leontina (Turner, 1892) & NP & & $\mathrm{X}$ & $\mathrm{X}$ & $\mathrm{X}$ \\
\hline Lecane ludwigi (Eckstein, 1883) & NP & & $\mathrm{X}$ & $\mathrm{X}$ & $\mathrm{X}$ \\
\hline Lecane luna (O. F. Müller, 1776) & NP & & & $\mathrm{X}$ & $\mathrm{X}$ \\
\hline Lecane lunaris Ehrenberg, 1832 & NP & & $\mathrm{X}$ & $\mathrm{X}$ & $\mathrm{X}$ \\
\hline Lecane monostyla (Daday, 1897) & $\mathrm{Pl} / \mathrm{NP}$ & & $\mathrm{X}$ & $\mathrm{X}$ & \\
\hline Lecane murrayi (Hauer, 1965) & NP & & & $\mathrm{X}$ & \\
\hline Lecane nodosa (Hauer, 1937/38) & NP & & & $\mathrm{X}$ & $\mathrm{X}$ \\
\hline Lecane obtusa (Murray, 1913) & NP & & & $\mathrm{X}$ & $\mathrm{X}$ \\
\hline Lecane opias Harring and Myers, 1926 & NP & & & & $\mathrm{X}$ \\
\hline Lecane papuana Murray, 1913 & NP & & $\mathrm{X}$ & $\mathrm{X}$ & $\mathrm{X}$ \\
\hline Lecane proiecta (Hauer, 1956) & NP & $\mathrm{X}$ & $\mathrm{X}$ & $\mathrm{X}$ & $\mathrm{X}$ \\
\hline Lecane pyriformis (Daday, 1905) & NP & & & $\mathrm{X}$ & $\mathrm{X}$ \\
\hline Lecane ruttneri Hauer, 1938 & NP & & & & $\mathrm{X}$ \\
\hline Lecane signifera (Jennings, 1896) & NP & & $\mathrm{X}$ & $\mathrm{X}$ & $\mathrm{X}$ \\
\hline Lecane stichaea Harring, 1913 & NP & & & $\mathrm{X}$ & $\mathrm{X}$ \\
\hline Lecane ungulata (Gosse, 1887) & NP & & & $\mathrm{X}$ & \\
\hline Lecane sp1. & NDT & & $\mathrm{X}$ & $\mathrm{X}$ & $\mathrm{X}$ \\
\hline Lecane sp2. & NDT & & $\mathrm{X}$ & $\mathrm{X}$ & $\mathrm{X}$ \\
\hline Lecane sp3. & NDT & & $\mathrm{X}$ & & $\mathrm{X}$ \\
\hline
\end{tabular}

$\mathrm{Pl}=$ planktonic, $\mathrm{NP}=$ not planktonic, $\mathrm{NDT}=$ non determined. 
Table 1. Continued...

\begin{tabular}{|c|c|c|c|c|c|}
\hline \multirow[b]{3}{*}{ Group } & \multirow[b]{3}{*}{ Habitat } & \multicolumn{4}{|c|}{ Environments and habitats } \\
\hline & & \multicolumn{2}{|c|}{ Island lakes } & \multicolumn{2}{|c|}{ Main channel } \\
\hline & & $\begin{array}{c}\text { Ilha } \\
\text { Grande }\end{array}$ & Pimental & Rapids & $\begin{array}{c}\text { Flow } \\
\text { restricted } \\
\text { waters }\end{array}$ \\
\hline \multicolumn{6}{|l|}{ COLURELLIDAE } \\
\hline Colurella uncinata (O.F. Muller, 1773) & NP & & $\mathrm{X}$ & $\mathrm{X}$ & \\
\hline Colurella sp. & NP & & $\mathrm{X}$ & $\mathrm{X}$ & $\mathrm{X}$ \\
\hline \multicolumn{6}{|l|}{ LEPADELLIDAE } \\
\hline Lepadella amphitropis Harring, 1916 & NP & & $\mathrm{X}$ & & $\mathrm{X}$ \\
\hline Lepadella benjamini Harring, 1916 & NP & & & & $\mathrm{X}$ \\
\hline Lepadella costata Wulfert, 1940 & NP & & $\mathrm{X}$ & & \\
\hline Lepadella cristata (Rousselet, 1893) & NP & $\mathrm{X}$ & & & \\
\hline Lepadella donneri Koste, 1972 & NP & & & & $\mathrm{X}$ \\
\hline Lepadella elliptica (Turner, 1892) & NP & & $\mathrm{X}$ & & \\
\hline Lepadella latusinus Myers, 1934 & NP & & $\mathrm{X}$ & $\mathrm{X}$ & $\mathrm{X}$ \\
\hline Lepadella patella (O. F. Müller, 1786) & NP & $\mathrm{X}$ & $\mathrm{X}$ & $\mathrm{X}$ & $\mathrm{X}$ \\
\hline Lepadella rhomboides (Gosse, 1886) & NP & & $\mathrm{X}$ & & $\mathrm{X}$ \\
\hline Lepadella sp. & NDT & & $\mathrm{X}$ & $\mathrm{X}$ & $\mathrm{X}$ \\
\hline Paracolurella logima (Myers, 1934) & NP & & $\mathrm{X}$ & & \\
\hline \multicolumn{6}{|l|}{ TRICHOTRIIDAE } \\
\hline Macrochaetus collinsi (Gosse, 1867) & NP & & $\mathrm{X}$ & $\mathrm{X}$ & $\mathrm{X}$ \\
\hline Macrochaetus sericus (Thorpe, 1893) & NP & & & $\mathrm{X}$ & \\
\hline Macrotrachela zickendrahti (Richters, 1902) & NDT & & & & $\mathrm{X}$ \\
\hline Trichotria tetractis (Ehrenberg, 1830) & NDT & & $\mathrm{X}$ & $\mathrm{X}$ & $\mathrm{X}$ \\
\hline Trichotria sp. & NDT & $\mathrm{X}$ & & & \\
\hline \multicolumn{6}{|l|}{ NOTOMATIDAE } \\
\hline Monommata arndti Remane, 1933 & NP & & $\mathrm{X}$ & & \\
\hline Monommata maculata Harring and Myers, 1924 & NP & & $\mathrm{X}$ & & \\
\hline Eosphora anthadis (Harring and Myers, 1922) & NP & & $\mathrm{X}$ & $\mathrm{X}$ & \\
\hline \multicolumn{6}{|l|}{ MYTILINIDAE } \\
\hline Mytilina macrocera (Jennings, 1894) & NP & & & & $\mathrm{X}$ \\
\hline Mytilina mucronata (Müller, 1773) & NP & & & $\mathrm{X}$ & \\
\hline Mytilina ventralis (Ehrenberg, 1832) & $\mathrm{N}$ & & $\mathrm{X}$ & & \\
\hline Mytilina sp. & NDT & & $\mathrm{X}$ & $\mathrm{X}$ & $\mathrm{X}$ \\
\hline \multicolumn{6}{|l|}{ SYNCHAETIDAE } \\
\hline Ploesoma lenticulare Herrick, 1885 & $\mathrm{Pl} / \mathrm{NP}$ & & $\mathrm{X}$ & & \\
\hline Ploesoma sp. & $\mathrm{Pl} / \mathrm{NP}$ & & $\mathrm{X}$ & & \\
\hline Polyarthra remata Skorikov, 1896 & $\mathrm{Pl}$ & $\mathrm{X}$ & $\mathrm{X}$ & & $\mathrm{X}$ \\
\hline Polyarthra vulgaris Carlin, 1943 & $\mathrm{Pl}$ & $\mathrm{X}$ & $\mathrm{X}$ & $\mathrm{X}$ & $\mathrm{X}$ \\
\hline Proales sp. & $\mathrm{Pl}$ & $\mathrm{X}$ & $\mathrm{X}$ & $\mathrm{X}$ & $\mathrm{X}$ \\
\hline Synchaeta stylata Wierzejski, 1893 & NP & $\mathrm{X}$ & & $\mathrm{X}$ & \\
\hline Synchaeta sp. & NP & & & $\mathrm{X}$ & \\
\hline \multicolumn{6}{|l|}{ PROALIDAE } \\
\hline Ptygura libera Myers, 1934 & NP & $\mathrm{X}$ & $\mathrm{X}$ & $\mathrm{X}$ & $\mathrm{X}$ \\
\hline Ptygura melicerta Ehrenberg, 1832 & NP & & & $\mathrm{X}$ & $\mathrm{X}$ \\
\hline Ptygura sp. & NP & & $\mathrm{X}$ & & $\mathrm{X}$ \\
\hline \multicolumn{6}{|l|}{ FLOSCULARIIDAE } \\
\hline Sinantherina sp. & NDT & & & & \\
\hline \multicolumn{6}{|l|}{ TESTUDINELLIDAE } \\
\hline Testudinela ahlstromi (Hauer, 1956) & NP & & $\mathrm{X}$ & $\mathrm{X}$ & $\mathrm{X}$ \\
\hline Testudinela ohlei (Koste, 1972) & NP & & $\mathrm{X}$ & $\mathrm{X}$ & $\mathrm{X}$ \\
\hline
\end{tabular}

$\mathrm{Pl}=$ planktonic, $\mathrm{NP}=$ not planktonic, $\mathrm{NDT}=$ non determined. 
Table 1. Continued...

\section{Group}

\begin{tabular}{ccccc} 
& \multicolumn{3}{c}{ Environments and habitats } \\
\cline { 2 - 5 } Habitat & \multicolumn{2}{c}{ Island lakes } & \multicolumn{2}{c}{ Main channel } \\
\cline { 2 - 5 } & $\begin{array}{c}\text { Ilha } \\
\text { Grande }\end{array}$ & Pimental & Rapids & $\begin{array}{c}\text { Flow } \\
\text { restricted } \\
\text { waters }\end{array}$ \\
\hline NP & & $\mathrm{X}$ & $\mathrm{X}$ & $\mathrm{X}$ \\
\hline NP & $\mathrm{X}$ & & $\mathrm{X}$ \\
\hline NP & $\mathrm{X}$ & $\mathrm{X}$ & $\mathrm{X}$ & $\mathrm{X}$ \\
NDT & & & & \\
\end{tabular}

\begin{tabular}{|c|c|c|c|c|c|}
\hline & \multirow{2}{*}{$\frac{\text { waters }}{\mathrm{X}}$} \\
\hline Testudinela patina (Hermann, 1783) & NP & & $X$ & $\mathrm{X}$ & \\
\hline Testudinela semiparva (Ternetz, 1892) & NP & & $\mathrm{X}$ & & \\
\hline Testudinela tridentata Smirnov, 1931 & NP & & & $\mathrm{X}$ & $\mathrm{X}$ \\
\hline Testudinela sp. & NDT & $\mathrm{X}$ & $\mathrm{X}$ & $\mathrm{X}$ & $\mathrm{X}$ \\
\hline \multicolumn{6}{|l|}{ TRICHOCERCIDAE } \\
\hline Trichocerca agnatha Wulfert, 1939 & NP & $\mathrm{X}$ & & & \\
\hline Trichocerca bicristata (Gosse, 1887) & NP & $\mathrm{X}$ & $\mathrm{X}$ & $\mathrm{X}$ & $\mathrm{X}$ \\
\hline Trichocerca bidens (Lucks, 1912) & NP & & $\mathrm{X}$ & $\mathrm{X}$ & $\mathrm{X}$ \\
\hline $\begin{array}{l}\text { Trichocerca capucina Wierzejski and Zacharias, } \\
1893\end{array}$ & NP & $\mathrm{X}$ & $\mathrm{X}$ & $\mathrm{X}$ & $\mathrm{X}$ \\
\hline Trichocerca chattoni (Beauchamp, 1907) & NP & $\mathrm{X}$ & $\mathrm{X}$ & $\mathrm{X}$ & $\mathrm{X}$ \\
\hline Trichocerca collaris (Rousselet, 1896) & NP & & $X$ & $\mathrm{X}$ & $\mathrm{X}$ \\
\hline Trichocerca gracilis (Tessin, 1890) & NP & $\mathrm{X}$ & $X$ & $\mathrm{X}$ & \\
\hline Trichocerca insiginis (Herrich, 1885) & NP & $\mathrm{X}$ & $X$ & $\mathrm{X}$ & $\mathrm{X}$ \\
\hline Trichocerca longiseta (Schrank, 1802) & $\mathrm{Pl}$ & & $\mathrm{X}$ & $\mathrm{X}$ & $\mathrm{X}$ \\
\hline Trichocerca myersi (Hauer, 1931) & NP & $\mathrm{X}$ & & $\mathrm{X}$ & $\mathrm{X}$ \\
\hline Trichocerca pusilla (Lauterborn, 1898) & NP & $\mathrm{X}$ & $\mathrm{X}$ & $\mathrm{X}$ & $\mathrm{X}$ \\
\hline Trichocerca rousseleti (Voigt, 1901) & NP & & $X$ & & \\
\hline Trichocerca similis (Wierzejski, 1893) & $\mathrm{Pl}$ & $\mathrm{X}$ & $X$ & $\mathrm{X}$ & $\mathrm{X}$ \\
\hline Trichocerca stylata (Gosse, 1851) & NP & $\mathrm{X}$ & & $\mathrm{X}$ & \\
\hline Trichocerca tigris (O.F.M., 1786) & NP & & & $\mathrm{X}$ & \\
\hline Trichocerca sp1. & $\mathrm{Pl} / \mathrm{NP}$ & $\mathrm{X}$ & & $\mathrm{X}$ & $\mathrm{X}$ \\
\hline Trichocerca $\mathrm{sp} 2$. & $\mathrm{Pl} / \mathrm{NP}$ & & $\mathrm{X}$ & $\mathrm{X}$ & $\mathrm{X}$ \\
\hline ORDEM BDELLOIDEA & NP & $\mathrm{X}$ & $\mathrm{X}$ & $\mathrm{X}$ & $\mathrm{X}$ \\
\hline \multicolumn{6}{|l|}{ CLADOCERA } \\
\hline \multicolumn{6}{|l|}{ CHYDORIDAE } \\
\hline Acroperus sp. & NP & & & & $\mathrm{X}$ \\
\hline Alona cambouei Guerne and Richard, 1893 & NP & & & $\mathrm{X}$ & \\
\hline Alona guttata Sars, 1862 & NP & & & $\mathrm{X}$ & \\
\hline Alona poppei Richard, 1897 & NP & & & $\mathrm{X}$ & $\mathrm{X}$ \\
\hline Alona sp. & NP & & & $\mathrm{X}$ & $\mathrm{X}$ \\
\hline Alonella dadayi Birge, 1910 & NP & & $\mathrm{X}$ & $\mathrm{X}$ & $\mathrm{X}$ \\
\hline Alonella sp1. & NP & & $\mathrm{X}$ & $\mathrm{X}$ & \\
\hline Alonella sp2. & NP & & & & $\mathrm{X}$ \\
\hline Disparalona hamata (Birge, 1879) & NP & & & & \\
\hline Pleuroxus sp. & NP & & & $\mathrm{X}$ & \\
\hline \multicolumn{6}{|l|}{ BOSMINIDAE } \\
\hline Bosmina hagmanni Stingelin, 1904 & P1 & $\mathrm{X}$ & & $\mathrm{X}$ & $\mathrm{X}$ \\
\hline Bosmina longirostris (O.F. Mueller, 1785) & $\mathrm{Pl}$ & $\mathrm{X}$ & $\mathrm{X}$ & $\mathrm{X}$ & $\mathrm{X}$ \\
\hline Bosminopsis deitersi Richard, 1834 & $\mathrm{Pl}$ & $\mathrm{X}$ & $\mathrm{X}$ & $\mathrm{X}$ & $\mathrm{X}$ \\
\hline \multicolumn{6}{|l|}{ DAPHNIDAE } \\
\hline Ceriodaphnia cornuta (Sars, 1886) & $\mathrm{Pl}$ & $\mathrm{X}$ & & & $\mathrm{X}$ \\
\hline \multicolumn{6}{|l|}{ ILYOCRYPTIDAE } \\
\hline Ilyocryptus spinifer (Herrich, 1884) & NP & & & $\mathrm{X}$ & $\mathrm{X}$ \\
\hline MACROTHRICIDAE & & & & & \\
\hline
\end{tabular}

$\mathrm{Pl}=$ planktonic, $\mathrm{NP}=$ not planktonic, $\mathrm{NDT}=$ non determined. 
Table 1. Continued...

\begin{tabular}{|c|c|c|c|c|c|}
\hline \multirow[b]{3}{*}{ Group } & \multirow[b]{3}{*}{ Habitat } & \multicolumn{4}{|c|}{ Environments and habitats } \\
\hline & & \multicolumn{2}{|c|}{ Island lakes } & \multicolumn{2}{|c|}{ Main channel } \\
\hline & & $\begin{array}{c}\text { Ilha } \\
\text { Grande }\end{array}$ & Pimental & Rapids & $\begin{array}{c}\text { Flow } \\
\text { restricted } \\
\text { waters }\end{array}$ \\
\hline Macrothrix spinosa King, 1853 & NP & & & $\mathrm{X}$ & \\
\hline Macrothrix superculeata Baird, 1843 & NP & $\mathrm{X}$ & & $\mathrm{X}$ & $\mathrm{X}$ \\
\hline Macrothrix sp1. & NP & & & & \\
\hline Macrothrix sp2. & NP & & & & $\mathrm{X}$ \\
\hline \multicolumn{6}{|l|}{ MOINIDAE } \\
\hline Moina minuta Hansen, 1899 & $\mathrm{Pl}$ & $\mathrm{X}$ & & & $\mathrm{X}$ \\
\hline \multicolumn{6}{|l|}{ COPEPODA } \\
\hline Copepodito & $\mathrm{Pl}$ & $\mathrm{X}$ & $\mathrm{X}$ & $\mathrm{X}$ & $\mathrm{X}$ \\
\hline Náuplio & $\mathrm{Pl}$ & $\mathrm{X}$ & $\mathrm{X}$ & $\mathrm{X}$ & $\mathrm{X}$ \\
\hline \multicolumn{6}{|l|}{ CALANOIDA } \\
\hline \multicolumn{6}{|l|}{ DIAPTOMIDAE } \\
\hline Argyrodiaptomus sp. & $\mathrm{Pl}$ & $\mathrm{X}$ & & $\mathrm{X}$ & \\
\hline Notodiaptomus sp1. & $\mathrm{Pl}$ & $\mathrm{X}$ & $\mathrm{X}$ & $\mathrm{X}$ & $\mathrm{X}$ \\
\hline Notodiaptomus sp2. & $\mathrm{Pl}$ & & $\mathrm{X}$ & $\mathrm{X}$ & $\mathrm{X}$ \\
\hline \multicolumn{6}{|l|}{ CYCLOPOIDA } \\
\hline \multicolumn{6}{|l|}{ CYCLOPIDAE } \\
\hline Thermocyclops sp1. & $\mathrm{Pl}$ & $\mathrm{X}$ & & & \\
\hline Thermocyclops sp2. & $\mathrm{Pl}$ & & & $\mathrm{X}$ & \\
\hline \multicolumn{6}{|l|}{ OTHER GROUPS } \\
\hline \multicolumn{6}{|l|}{ PROTOZOA } \\
\hline \multicolumn{6}{|l|}{ CENTROPYXIDAE } \\
\hline Centropyxis spp. & & & $\mathrm{X}$ & $\mathrm{X}$ & $\mathrm{X}$ \\
\hline \multicolumn{6}{|l|}{ EUGLYPHIDAE } \\
\hline Euglypha spp. & & $\mathrm{X}$ & $\mathrm{X}$ & $\mathrm{X}$ & $\mathrm{X}$ \\
\hline \multicolumn{6}{|l|}{ VORTICELLIDAE } \\
\hline Vorticella spp. & & $X$ & $\mathrm{X}$ & $\mathrm{X}$ & $\mathrm{X}$ \\
\hline OSTRACODA & & & $\mathrm{X}$ & $\mathrm{X}$ & $\mathrm{X}$ \\
\hline \multicolumn{6}{|l|}{ GASTROTRICHA } \\
\hline Família Chaetonotidae & & $\mathrm{X}$ & & & \\
\hline \multicolumn{6}{|l|}{ INSECTA } \\
\hline Acaro & & $\mathrm{X}$ & $\mathrm{X}$ & $\mathrm{X}$ & $\mathrm{X}$ \\
\hline Chaoboridae Famíly (larvae) & & $\mathrm{X}$ & $\mathrm{X}$ & $\mathrm{X}$ & $\mathrm{X}$ \\
\hline Chironomidae Famíly (larvae) & & & $X$ & $\mathrm{X}$ & $X$ \\
\hline Odonata & & $\mathrm{X}$ & $\mathrm{X}$ & $\mathrm{X}$ & $\mathrm{X}$ \\
\hline \multicolumn{6}{|l|}{ UNIONICOLIDAE } \\
\hline Unionicola spp. & & & $\mathrm{X}$ & $\mathrm{X}$ & \\
\hline
\end{tabular}

$\mathrm{Pl}=$ planktonic, $\mathrm{NP}=$ not planktonic, $\mathrm{NDT}=$ non determined.

tridentata Smirnov, 1931; Lecane murrayi (Hauer, 1965), and Ilyocryptus spinifer (Herrich, 1884) all occurred exclusively in the main channel, whereas Brachionus mirabilis (Daday, 1897), Gastropus stylifer Imhof, 1891; Trichocerca rousseleti (Voigt, 1901), and Macrothrix superculeata Baird, 1843 were found only in the lakes.

\section{Discussion}

The rotifers were the most diverse group of zooplankton recorded in the present study on the middle Xingu River in the southeastern Amazon basin, with approximately $80 \%$ of the organisms found in each study environment. A similar pattern has been recorded in the aquatic systems of 
Table 2. Number of genera and taxa within zooplankton groups for the midd Xingu river habitats.

\begin{tabular}{lcc}
\hline \multicolumn{1}{c}{ Group } & Genera & Taxa \\
\hline ROTIFERA & 38 & 141 \\
Monogononta & 38 & 141 \\
Bdelloidea & - & 1 \\
CLADOCERA & 11 & 20 \\
COPEPODA & 3 & 5 \\
Cyclopoida & 1 & 2 \\
Calanoida & 2 & 3 \\
Total & 52 & 166 \\
\hline
\end{tabular}

white- $(77 \%)$ and black-water (40\%) rivers in the Amazon basin (Table 3), as well as other systems (see Green, 1972; Dumont, 1983; Neves et al., 2003; Paggi and José de Paggi, 1990; Sampaio and López, 2000).

The distinct composition of the zooplankton found in the two lakes studied here reflect their different limnological characteristics, such as the much larger surface area of the Ilha Grande lake in comparison with Pimentel, which in turn is subject to a considerable input of organic matter from the surrounding forest, which also shelters the lake from the sun. Pimentel lake is also connected more extensively to the main river, with the water being

Table 3. Taxonomic richness for the zooplankton of different Amazon environments.

\begin{tabular}{|c|c|c|c|c|c|}
\hline Environment & $\begin{array}{c}\text { Water } \\
\text { category }\end{array}$ & Rotifera & Cladocera & Copepoda & Main source \\
\hline Amazon-Solimões system & Whites & 110 & & 17 & Robertson and Hardy (1984) \\
\hline Calado Lake & Whites & & 8 & & Robertson and Hardy (1984) \\
\hline Camaleão Lake & Whites & 175 & & & Koste et al. (1984) \\
\hline Castanho Lake & Whites & & 16 & & Robertson and Hardy (1984) \\
\hline Jacaretinga Lake & Whites & & 12 & & Robertson and Hardy (1984) \\
\hline Redondo Lake & Whites & & 5 & & Robertson and Hardy (1984) \\
\hline Manacuri Lake & Whites & & 16 & & Robertson and Hardy (1984) \\
\hline Branco river & Whites & 11 & 1 & & Robertson and Hardy (1984) \\
\hline Madeira river & Whites & 60 & & 7 & Robertson and Hardy (1984) \\
\hline Maracá-Roraima Island & Whites & 159 & & & Koste and Robertson (1990) \\
\hline $\begin{array}{l}\text { Cuiabá river } \\
\text { marginal lakes }\end{array}$ & Whites & 79 & 30 & 6 & Neves et al. (2003) \\
\hline $\begin{array}{l}\text { Acre river } \\
\text { Amapá lake, Pirapora lake }\end{array}$ & Whites & 38 & 6 & 2 & Keppeler (2003) \\
\hline Amapá lake & Whites & 30 & 5 & 3 & Keppeler and Hardy (2004) \\
\hline Negro river & Blacks & 50 & 7 & 18 & Robertson and Hardy (1984) \\
\hline Cristalino lake & Blacks & & 6 & & Robertson and Hardy (1984) \\
\hline Tarumã-Mirim lake & Blacks & & 12 & & Robertson and Hardy (1984) \\
\hline Guedes lake & Blacks & & 7 & & Robertson and Hardy (1984) \\
\hline Caju lake & Blacks & & 5 & & Robertson and Hardy (1984) \\
\hline Prato lake & Blacks & & 3 & & Robertson and Hardy (1984) \\
\hline $\begin{array}{l}\text { Utinga-Pará system } \\
\text { Bolonha lake }\end{array}$ & Blacks & 30 & 19 & 7 & Melo et al. (2006) \\
\hline Tapajós river & Clears & 127 & & 8 & Robertson and Hardy (1984) \\
\hline Paroni lake & Clears & 76 & & & Koste (1974a) \\
\hline Tocantins river & Clears & 21 & $5-7$ & 14 & Robertson and Hardy (1984) \\
\hline Tauá lake & Clears & 6 & & & Robertson and Hardy (1984) \\
\hline Paulo pool & Clears & 3 & & & Robertson and Hardy (1984) \\
\hline Lower Nhamundá river & Clears & 145 & & & Brandorff et al. (1982) \\
\hline $\begin{array}{l}\text { Trombetas river } \\
\text { Macaco lake }\end{array}$ & Clears & 48 & & & Koste (1989) \\
\hline $\begin{array}{l}\text { Batata lake (impacted by } \\
\text { bauxite waste) }\end{array}$ & Clears & 98 & 10 & 7 & Bozelli et al. (2000) \\
\hline $\begin{array}{l}\text { Xingu river } \\
\text { (lentic waters / rapids) }\end{array}$ & Clears & $55-87$ & $10-16$ & $1-2$ & This study \\
\hline Ilha Grande lake & Clears & 56 & 6 & 3 & This study \\
\hline Pimental kake & Clears & 97 & 5 & 4 & This study \\
\hline
\end{tabular}


filtered by the surrounding forest before draining into the lake (Estupiñan and Camargo, 2008). The considerable phytoplankton biomass and high primary productivity recorded in Ilha Grande lake (Costa et al., 2008) may have determined the low zooplankton richness recorded in this lake. The higher zooplankton diversity recorded in Pimentel lake appears to have been related to specific characteristics of this environment, such as the low current velocity, given that reproductive populations of planktonic organisms are restricted to the slow-flowing lower reaches of these areas (Ward, 1994).

\section{Acknowledgements}

The present study was supported by the project "A trophic model for the management of the middle Xingu River", financed by ANEL. The authors wish to thank the anonymous reviewers for comments on an earlier version of this paper.

\section{References}

BOZELLI, RL., 1992. Composition of the zooplankton community of Batata and Mussurá lakes and of the Trombetas River, State of Pará, Brasil. Amazoniana, vol. 12, p. 239-261.

BOZELLI, RL., 1994. Zooplankton community density in relation to water level fluctuation and inorganic turbity in an Amazonian lake, Lago Batata State of Pará Brazil. Amazoniana, vol. 13, p. 17-32.

BOZELLI, RL., ESTEVES, FA. and ROLAND, F., 2000. Lago Batata: impacto e recuperação de um ecossistema amazônico. Rio de Janeiro: Instituto de Biologia UFRJ/Sociedade Brasileira de Limnologia. 265 p.

BRANDORFF, GO. and ANDRADE, ER., 1978. The relationship between the water level of the Amazon River and fate of the zooplankton population in Lago Jacaretinga, a Várzea lake in Central Amazon. Studies on Neotropical Fauna and Environment, vol. 13, no. 2, p. 63-70. http://dx.doi.org/10.1080/01650527809360533.

BRANDORFF, GO., 1973. Neue freilebende Calanoide Copepoden (Crustacea) aus den Amazonasgebiet. Amazoniana, vol. 4, p. 205-218.

BRANDORFF, GO., KOSTE, W. and SMIRNOV, NV., 1982. The composition and structure of Rotiferan and Crustacean communities of the lower Rio Nhamundá, Amazonas, Brazil. Studies on Neotropical Fauna and Environment, vol. 1, no. 2-3, p. 69-121. http://dx.doi.org/10.1080/01650528209360604.

CAMARGO, M., GIARRIZZO, T. and ISAAC, V., 2005. Review of the Geographic distribution of fish fauna of the Xingu River basin, Brazil. Ecotropica, vol. 10, p. 123-147.

CARVALHO, ML., 1983. Efeitos da flutuação do nível da água sobre a densidade e composição do zooplâncton em um lago de várzea da Amazônia, Brasil. Acta Amazonica, vol. 13, p. 715-724.

CIPÓLLI, MN. and CARVALHO, MAJ., 1973. Levantamento de Calanoida e Cyclopoida (Copepoda, Crustacea) das águas da região do Guamá, Capim e Tocantins, com nota sobre a fauna acompanhante. Papéis Avulsos de Zoologia, vol. 27, p. 95-110.

COSTA, V., COSTA, S. and CAMARGO, M., 2008. Os produtores primários: o fitoplâncton e o Periliton. In CAMARGO, M. and GHILHARDI, R. (Eds.). Entre a terra, as águas e os pescadores do médio rio Xingu: uma abordagem ecológica. Belém: Maurício Camargo. 329 p.

CRITCHFIELD, HJ., 1968. General climatology. Nova Delhi: Prentice-Hall. 420 p.

DUMONT, HJ., 1983. Biogeography of rotifers. Hydrobiologia, vol. 104, no. 1, p. 19-30. http://dx.doi.org/10.1007/BF00045948.

ELMOOR-LOUREIRO, LM., 1997. Manual de identificação de cladóceros límnicos do Brasil. Brasília: Universa. 156 p.

ESTUPIÑAN, R. and CAMARGO, M., 2008. Ecologia da paisagem natural. In CAMARGO, M. and GHILHARDI, R. (Eds.). Entre a terra, as águas e os pescadores do médio rio Xingu: uma abordagem ecológica. Belém: Maurício Camargo. 329 p.

FERNANDO, CH., 2002. A guide to tropical freshwater zooplankton: identification, ecology and impact on fisheries. Leiden: Backhuys Publishers. 291 p.

GREEN, J., 1972. Freshwater ecology in the Mato Grosso, Central Brazil. III. Associations of Rotifera in meander lakes of the Rio Suiá Missú. Journal of Natural History, vol. 6, no. 2, p. 229-241. http://dx.doi.org/10.1080/00222937200770221.

HARDY, ER., 1980. Composição do zooplâncton em cinco lagos da Amazônia Central. Acta Amazonica, vol. 10, p. 577-609.

HARDY, ER., ROBERTSON, BA. and KOSTE, W., 1984. About relationship between the zooplankton and fluctuating water levels of Lago Camaleão, a Central Amazonian várzea lake. Amazoniana, vol. 9, p. 43-52.

KEPPELER, EC. and HARDY, ER., 2004. Abundance and composition of Rotifera in an abandoned meander lake (Lago Amapá) in Rio Branco, Acre, Brazil. Revista Brasileira de Zoologia, vol. 21, no. 2, p. 233-241. http://dx.doi.org/10.1590/ S0101-81752004000200011.

KEPPELER, EC., 2003. Comparative study of the zooplankton composition of two lacustrine ecosystems in southwestern Amazonia. Acta Scientiarum, vol. 25, p. 471-481.

KOROVCHINSKY, NM., 1992. Sididae and Holopedidae: (Crustacea: Daphniiformes). The Hague: SPB Academic Publishing. $82 \mathrm{p}$.

KOSTE, W. and ROBERTSON, B., 1983. Taxonomic studies of the Rotifera (Phylum Aschelminthes) from a Central Amazonian varzea lake, Lago Camaleão (Ilha de Marchantaria, Rio Solimões, Amazonas, Brazil). Amazoniana, vol. 8, p. 225-254.

KOSTE, W. and ROBERTSON, B., 1990. Taxonomic studies of rotifera from shallow waters on the Island of Maracá, Roraima, Brazil. Amazoniana, vol. 11, p. 185-200.

KOSTE, W., 1972. Rotatorien aus Gewässern Amazonies. Amazoniana, vol. 3, p. 258-505.

KOSTE, W., 1974a. Rotatorien aus einem Ufersee des unteren Rio Tapajós, dem Lago Paroni (Amazonien). Gewäs. u. Abwäs., vol. 53-54, p. 43-68.

KOSTE, W., 1974b. Zur Kenntnis der Rotatorienfauna der "Schwimmenden Wiese" einer Uferlagune in der Varzea Amazoniens, Brasilien. Amazoniana, vol. 5, p. 25-60.

KOSTE, W., 1978. Rotatotia Die Rodertiere Mitteleuropes bëgründet von Max Voigt-Monogononta. 2. Auflage neubearbeitet von Water Koste. Berlin: Gebrüder Borntrager. vol. 1, 673 p. 
KOSTE, W., 1989. Über Rädertiere (Rotatoria) aus dem Lago do Macaco, einem Ufersee des mittleren Rio Trombetas. Osnabrücker naturwissenschaftliche Mitteilungen, vol. 15, p. 190-214.

KOSTE, W., ROBERTSON, BA. and HARDY, E., 1984. Further taxonomical studies of the rotifera from Lago Camaleão. A central amazonian várzea lake (Ilha de Marchantaria, Rio Solimões, Brazil). Amazoniana, vol. 8, p. 555-576.

MAGAlHÃES, C., MALTA, JC., ROBERTSON, BA. and VARELLA, A., 1988. A catalogue of type specimens of crustacea in the invertebrate collection of the Instituto Nacional de Pesquisas da Amazonia, INPA, Manaus, Brazil, up to January 1988. Amazoniana, vol. 10, p. 267-282.

MELO, NFAC., PAIVA, RS. and SILVA, MMT., 2006. Considerações ecológicas sobre o zooplâncton do lago Bolonha, Belém, Pará, Brasil. Boletim do Museu Paraense Emílio Goeldi. Ciências Naturais, vol. 1, p. 115-125.

NEVES, IF., ROCHA, O., ROCHE, KF. and PINTO, AA., 2003. Zooplankton community structure of two marginal lakes of the River Cuiabá (Mato Grosso, Brazil) with analysis of Rotifera and Cladocera Diversity. Brazilian Journal of Biology, vol. 63, no. 2, p. 329-343. http://dx.doi.org/10.1590/S1519-69842003000200018. PMid:14509855.

PAGGI, JC. and JOSÉ DE PAGGI, S., 1990. Zooplankton from lotic and lentic environments of the middle River Paraná. Acta Limnologica Brasiliensia, vol. 3, p. 685-719.

PAGGI, JC., 1979. Revisión de las especies argentinas del genero Bosmina Baird agrupadas en el subgênero Neosbomina Lieder (Crustacea, Cladocera). Acta Zoológica Lilloana, vol. 35, p. 137-162.

PAGGI, JC., 1995. Crustacea Cladocera. In LOPRETTO, EC. and TELL, G. (Eds.). Ecosistemas de aguas continentales: metodologias para su estudio III. La Plata: Ediciones Sur. p. 909-971.

REID, JW., 1985. Chave de identificação e lista de referências bibliográficas para as espécies continentais sulamericanas de vida livre da ordem Cyclopoida (Crustacea, Copepoda). Boletim de Zoologia da Universidade de São Paulo, vol. 9, p. 17-143.

ROBERTSON, BA. and HARDY, ER., 1984. Zooplankton of Amazonian lakes and rivers. In SIOLI, H. (Ed.). The Amazon: limnology and landscape. The Hague: Dr. W. Junk Publishers. p. 337-352. Monographie Biologicae, vol. 56. http://dx.doi. org/10.1007/978-94-009-6542-3_13.

ROBERTSON, BA., 1980. Composição, abundância e distribuição de Cladocera (Crustacea) na região de água livre da represa de Curuá-Una, Pará. Manaus: Fundação Universidade do Amazonas, Instituto Nacional de Pesquisas da Amazônia. 105 p. Master's Dissertation.

ROBERTSON, BA., SILVA, ENS. and REID, J., 1989. Atlas de copépodos planctônicos Calanoida e Cyclopoida (Crustacea) da Amazônia Brasileira. I. Represa de Curuá-Una, Pará. Revista Brasileira de Zoologia, vol. 6, p. 725-758.

SAMPAIO, EV. and LÓPEZ, CM., 2000. Zooplankton community composition and some limnological aspects of an oxbow lake of the Paraopeba River, São Francisco River Basin, Minas Gerais, Brazil. Brazilian Archives of Biology and Technology, vol. 43, no. 3, p. 285-293. http://dx.doi.org/10.1590/S1516-89132000000300007.

SANTOS-SILVA, EN., ROBERTSON, BA., REID, J. and HARDY, ER., 1989. Atlas de copépodos planctônicos, Calanoida e Cyclopoida (Crustacea), da Amazônia Brasileira. Volume I. Represa de Curuá-Una, Pará. Revista Brasileira de Zoologia, vol. 6 , p. $725-758$

SEGERS, H., 1995. Rotifera: the Lecanidae (Monogononta). Amsterdam: SPB Academic Publishing. vol. 2, 226 p.

SMIRNOV, NN., 1992. The Macrothricidae of the world III. (Guides to the identification of the microinvertebrates of the continental waters of the world). Amsterdam: SPB Academic Publishing. $143 \mathrm{p}$.

WAICHMAN, AV., GARCÍA-DÁVILA, CR., HARDY, ER. and ROBERTSON, BA., 2002. Composição do zooplâncton em diferentes ambientes do lago Camaleão, na ilha da Marchantaria, Amazonas, Brasil. Acta Amazonica, vol. 32, no. 2, p. 339-347. http://dx.doi.org/10.1590/1809-43922002322347.

WARD, JV., 1994. The structure and dynamics of lotic ecosystems. In MARGALEF, R. (Ed.). Limnology now: a paradigm of planetary problems. Amsterdam: Elsevier Science BV. 218 p. 